Vol. 19, Núm. 1, 2017

\title{
Los profesores frente a la nueva/vieja escuela secundaria argentina ${ }^{1}$
}

\section{Teachers and the New/Old Argentinean Secondary School}

\author{
Lea Fernanda Vezub (*) leitiv@gmail.com \\ María Florencia Garabito (*) florenciagarabito@gmail.com \\ (*) Universidad Nacional de Moreno \\ (Recibido: 20 de febrero de 2015; Aceptado para su publicación: 22 de octubre de 2015)
}

Cómo citar: Vezub, L. F. y Garabito, M. F. (2017). Los profesores frente a la nueva/vieja escuela secundaria argentina. Revista Electrónica de Investigación Educativa, 19(1), 123-140. Recuperado de https://doi.org/10.24320/redie.2017.19.1.1096

\section{Resumen}

El nivel secundario es actualmente objeto de múltiples transformaciones, políticas y debates. Se cuestiona su función histórica, la organización del currículum, el trabajo de los docentes y de qué manera es posible dar sentido a la experiencia escolar de los adolescentes. El artículo presenta los resultados de un proyecto de investigación, cuyo propósito fue indagar cómo perciben estos cambios los profesores, cuáles son los problemas que identifican y qué reposicionamientos se generan en sus identidades profesionales. A tal fin se consideraron los procesos de inclusión de nuevos adolescentes y la integración de las TIC en la secundaria, y se analizan los desafíos para la formación y desarrollo profesional de los docentes. La investigación se llevó a cabo desde una perspectiva sociocrítica; con métodos de recolección de información en extensión y la aplicación de un cuestionario semi-estructurado. Las preguntas abiertas fueron sistematizadas con el software Atlas.ti para análisis de datos cualitativos.

Palabras clave: Secundaria, formación docente, formación profesional, democratización de la educación.

\begin{abstract}
Secondary education is currently the subject of many transformations, policies and debates. These call into question its historical function, the organization of its curriculum, teachers' work,

\footnotetext{
${ }^{1}$ La denominación de "nueva escuela secundaria" se refiere al proceso de transformación que este nivel educativo atraviesa en Argentina y surge con posterioridad a las reformas de los años noventa, ya que se enmarca en la última Ley Nacional de Educación (No 26206 de 2006). El marco normativo y lineamientos generales de esta "nueva escuela secundaria" -tal como es nombrada en documentos- se fija a partir de las Resoluciones No84 y No88 de 2009 del Consejo Federal de Educación que establecen Lineamientos Políticos y Estratégicos de la Educación Secundaria Obligatoria, por una parte y el fortalecimiento de las instituciones a través de los Planes Jurisdiccionales de mejora institucional. Resoluciones posteriores fijan la necesidad de brindar acompañamiento a las trayectorias escolares y de aprendizaje de los estudiantes para lograr una escuela inclusiva y políticas educativas que garanticen el derecho a la educación y la inclusión, es decir el efectivo acceso, la continuidad escolar y el egreso; así como de las condiciones pedagógicas y materiales para hacer posible el tránsito por la secundaria, en tanto nivel obligatorio del sistema educativo.
} 
and the way it gives meaning to adolescents' academic experience. This paper offers the results of a research project that sought to determine how teachers perceive these changes, the problems they identify, and how their professional identities may be repositioned as a result. With that in mind, the study considered the inclusion processes for new adolescents and ICT integration in secondary schools, and analyzes the challenges in teacher training and professional development. The study was carried out from a sociocritical perspective, and applied extended information collection methods and a semi-structured questionnaire. The open-ended questions were systematized with the software Atlas.ti for qualitative data analysis.

Keywords: Secondary school teacher, teacher education, professional training, democratization of education.

\section{Introducción}

No es novedad afirmar que la escuela secundaria se presenta en América Latina como el nivel más crítico y polémico cuando se piensa en cómo producir los cambios deseados, qué reformas son necesarias y cómo planificar políticas para su mejoramiento. Si se observa la cotidianidad de las instituciones, se aprecia que se han alterado de manera profunda una serie de prácticas y supuestos que hacían posible el orden escolar y el vínculo entre docentes y estudiantes. La comunicación pedagógica y la "recepción de los mensajes" no están aseguradas por la institución (Corea, 2005). La tarea de los docentes, la enseñanza y la propia función de la escuela se encuentran en constante cuestionamiento. Ello demanda un nuevo tipo de identidad profesional, otros saberes, actitudes, competencias y sensibilidades.

Para Dubet (2006) se trata de un oficio que sus actores perciben como "imposible", en tanto ya no responde a los cánones que fija el imaginario colectivo de la profesión. La identidad del profesorado, construida tanto a la largo de la formación inicial, como durante la propia biografía escolar de los enseñantes y, posteriormente, actualizada en las instancias del perfeccionamiento, presenta numerosos puntos de ruptura con las exigencias actuales que hoy implica la docencia. Los programas y acciones de capacitación en servicio generalmente se han centrado en los contenidos de la disciplina y a veces en su enseñanza, escindiendo la formación de las características de los sujetos de aprendizaje y de los escenarios reales de desempeño, de las problemáticas cotidianas que enfrentan las escuelas. Razones por las cuales, la formación continua ha contribuido poco a la revisión de las identidades profesionales del profesorado secundario y a su puesta en diálogo con los demandas del oficio, en los nuevos escenarios de la escolaridad (Vezub, 2011).

Los comentarios de los docentes ponen en evidencia que tanto viejos como nuevos profesores no se sienten preparados para trabajar, enseñar e interactuar con la subjetividad de los alumnos en las aulas (Alliaud y Antelo, 2008; Vezub, 2011). No se trata sólo de la falta de estrategias didácticas, de supuestas fallas o lagunas en la formación inicial, sino de códigos, escenarios y territorios diferentes, intensamente transmutados que se perciben como abismos culturales. La brecha entre el estatus conferido y asignado al trabajo de los profesores secundario y el ejercicio real, el oficio tal-cual es actuado en las instituciones- se ha agrandado cada vez más.

La comentada crisis de la escuela secundaria o el declive del programa institucional (Dussel, 2007; Bolívar, León y Pérez, 2005; Dubet, 2006) deben interpretarse, en primer lugar, en el contexto de la progresiva extensión de la obligatoriedad escolar; es decir, de los efectos que ocasiona la incorporación de nuevos públicos y sectores, antes excluidos del nivel secundario del sistema educativo. En segundo lugar, no son menores las consecuencias que tienen los cambios acelerados en las formas de producir, circular e intercambiar conocimiento, cultura y bienes en las sociedades posmodernas. Aquí debe ubicarse la disputa que la escuela mantiene como espacio de trasmisión cultural y constitución de la subjetividad, con los discursos mediáticos y las TIC (Rascován, 2013). 
En este escenario realizamos una investigación con sede en el Departamento de Humanidades de la Universidad Nacional de Moreno (Argentina), cuyo objetivo fue analizar las transformaciones recientes que los docentes experimentan en la tarea y oficio de enseñar. Particularmente, recortamos como emergentes dos políticas que actualmente se implementan en secundaria: la extensión de la obligatoriedad del nivel y la integración de las TIC en las aulas.

Una de las hipótesis de trabajo es que frente a estas políticas y cambios del nivel medio, la identidad profesional de los educadores se siente amenazada, produciéndose, en muchos casos, un "repliegue identitario" que se observa en actitudes defensivas y de auto-sostenimiento en sintonía con las identidades docentes tradicionales (Dussel, 2007; Brito, 2012) y fundantes del profesor secundario. Otros docentes, como se verá, se alejan de la victimización, asumen un posicionamiento diferente, intentan adaptarse a los cambios y comienzan a revisar críticamente las bases de su oficio, en búsqueda de una nueva identidad profesional (Bolívar, Gallego, León y Pérez, 2005). Sin embargo, todavía no se avizora cuáles son los sentidos hacia los que dichas identidades se desplazan en el marco de las políticas de inclusión educativa e integración de tecnologías; es por ello que el proyecto indagó los reposicionamientos y trasformaciones que empiezan a vislumbrase en las identidades y trayectorias de los profesores. ${ }^{2}$

\section{Método}

Para el trabajo de campo se elaboró un cuestionario exploratorio que fue respondido por 118 profesores secundarios en dos instancias, la primera en noviembre de 2012 y, la segunda en abril de 2014. La mayoría de los encuestados pertenece a la localidad de Moreno (59\%) o áreas aledañas, tres cuartas partes son profesores del Conurbano Bonaerense.

A pesar de tratarse de una muestra casual o intencional, que no fue construida en base a parámetros estadísticos de representatividad, la distribución por sexo de los profesores es similar a la del último Censo Nacional de Docentes (publicado) para el nivel secundario: 63\% de mujeres y $36 \%$ de varones. ${ }^{3}$ El $86 \%$ tiene más de 40 años, mientras que sólo un $15 \%$ se ubicó entre los 20 y 30 años. Los entrevistados acumulan bastante antigüedad docente: un poco más de un tercio posee 16 años o más de experiencia en el cargo. Por el contrario, la tasa de nóveles es menor, los profesores con hasta 5 años de experiencia no llega a la cuarta parte (22\%). Es decir, que la mayoría ha experimentado en su carrera tanto cambios curriculares, como legislativos y estructurales que han incidido en sus trayectorias de formación y opciones profesionales.

Entre estos cambios, cabe mencionar La Ley Federal de Educación de 1993 que prolongó la obligatoriedad escolar hasta el primer ciclo de la escuela secundaria. En la década del noventa se introdujeron cambios estructurales en el sistema en un contexto de crisis económica y precarización del empleo. Se creó la Enseñanza General Básica (EGB) que abarcaba el último año de la primaria y los dos primeros de la secundaria, y el ciclo del Polimodal (secundaria propiamente dicha), con diversas orientaciones y trayectos técnicos profesionales. La EGB funcionó algunas veces en instituciones primarias y otras en articulación con establecimientos secundarios, produciendo diversos conflictos y una reconversión de los perfiles docentes. Se elaboraron nuevos parámetros y documentos curriculares a nivel nacional (Contenidos Básicos Comunes), se establecieron nuevos procedimientos de gestión pedagógica (la elaboración de Proyectos Educativos Institucionales, por ejemplo) y se promovió la capacitación masiva de los

\footnotetext{
${ }^{2}$ Se trató de una investigación breve y exploratoria que se propuso generar hipótesis para posteriores investigaciones en torno a los cambios recientes y al posicionamiento de los docentes. En consecuencia, los resultados plantean algunas hipótesis de trabajo aún provisorias sobre las identidades profesionales que se espera profundizar en los proyectos recientemente aprobados y en ejecución (PICT No. 1282-2014, FONCYT, Agencia Nacional de Promoción Científica y Tecnológica, Argentina).

${ }^{3}$ A nivel nacional se registra un $68.5 \%$ de docentes varones en ejercicio en nivel secundario y un $31.5 \%$ de profesoras mujeres (Fuente: Boletín No4, DINIECE, 2007. El perfil de los docentes en la Argentina. Datos del Censo Nacional de Docentes 2004, Ministerio de Educación).
} 
docentes en la actualización de los contenidos básicos comunes. Posteriormente, la Ley de Educación Nacional 26.026 sancionada en 2006 estableció como obligatorio todo el nivel secundario y retornó a la estructura del nivel primario y secundario. Asimismo comenzó un proceso de ordenamiento de las modalidades y orientaciones del ciclo superior de la secundaria que acota el número de opciones (Res. 84/2009, CFE) y lleva a la elaboración de nuevos diseños curriculares.

Por otro lado, en el 2009 se crearon los programas nacionales "Una computadora para cada alumno" (Res. CFE 82/2009) y "Conectar Igualdad" (Decreto 459/2010), mediante los cuales se entregó una notebook a cada profesor y estudiante de las escuelas secundarias estatales, del sistema de formación docente y de la educación especial; junto con la capacitación de los profesores, la elaboración de materiales y propuestas educativas para la incorporación de las TIC en los procesos de enseñanza y aprendizaje. Estos son algunos de los cambios que demandan una nueva profesionalidad en los docentes que participaron de este estudio.

En cuanto al sector de gestión de las instituciones, la amplia mayoría (59\%) enseña exclusivamente en escuelas públicas, estatales, y sólo un 14\% en instituciones privadas, mientras que casi un cuarto (23.7\%) tiene horas en ambos sectores. La mayoría (45\%) caracteriza la territorialidad de las instituciones como "periférica", se desempeñan en zonas alejadas del centro que reciben alumnos de población más vulnerable y menor nivel socioeconómico. En segundo lugar se encuentran quienes tienen sus horas en escuelas céntricas (36.5\%) y, por último, el 18.6\% trabaja en escuelas que califica como "muy periféricas". ${ }^{4}$ Los docentes consideran que la zona y el barrio de las instituciones inciden en su tarea, debido a las características sociales, valores y consumos culturales de los adolescentes y de las familias. Para casi la mitad el territorio tiene "mucha incidencia" (45\%), igual proporción opina que tiene "alguna incidencia", y un 10\% indica que la localización de la escuela no afecta su tarea. ${ }^{5}$

Prácticamente la mitad (44\%) de los entrevistados tiene horas en dos y tres establecimientos, mientras una quinta parte asiste a cuatro o cinco. ${ }^{6}$ Si se suman estas categorías, se obtiene que dos tercios de los profesores trabajan en dos a cinco escuelas, lo que muestra una sobrecarga importante de trabajo. En el mismo sentido, el estudio de Tenti (2005) establece que numerosos docentes trabajan en varias instituciones: en Argentina un 30\% de profesores secundarios se desempeña en dos establecimientos y el $11.5 \%$ en tres, lo que -según el autor- obstaculiza la pertenencia institucional y la posibilidad de trabajar con otros. El estrés y la tensión laboral aumentan cuando los profesores tienen que adaptarse a varias instituciones, a distintos equipos, estilos de conducción, normas institucionales y poblaciones estudiantiles.

De este modo, los datos de base de los profesores encuestados dan cuenta de una población docente atravesada e involucrada en las problemáticas que el proyecto se propuso investigar: la expansión de la escuela a nuevos sectores, el desafío de repensar las identidades profesionales y la tarea docente.

Se trabajó con un instrumento semi-estructurado (tres preguntas abiertas y las restantes cerradas) y una perspectiva metodológica sustentada en la teoría crítica reconceptualizada, que recoge la tradición sociocrítica y cualitativa de la investigación educativa (Kincheloe y McLaren,

\footnotetext{
${ }_{5}^{4}$ La suma es mayor al $100 \%$, ya que algunos profesores trabajan en más de un tipo de escuela.

${ }^{5} \mathrm{~A}$ modo de hipótesis planteamos que existe (en un grupo de los docentes argentinos) un supuesto acerca del docente homogéneo e intercambiable, capaz de enseñar en cualquier contexto sociocultural. La tarea de educar se entiende como única, al concebir que los mismos contenidos y estrategias para todas las poblaciones garantizan la calidad. En este sentido podría interpretarse la respuesta de los profesores al afirmar que la localización de las escuelas no afecta su trabajo docente, ya que éste sería independiente del contexto.

${ }^{6}$ En Argentina la organización que predomina para la contratación de los profesores es su designación por horas cátedra frente a alumnos, por lo que generalmente se produce una alta dispersión de profesores entre varios establecimientos, quienes deben tomar horas en distintas escuelas para reunir un salario equivalente al de un cargo docente promedio.
} 
2012). La investigación utilizó un diseño flexible, en interacción permanente entre el trabajo de campo, el análisis de los datos y la construcción -reconstrucción del problema y de los interrogantes junto a la discusión teórica (Rockwell, 2009).

Las preguntas abiertas se categorizaron a posteriori, a través de la lectura minuciosa de las respuestas y la realización de sucesivos agrupamientos con la ayuda del Atlas.ti, hasta que se obtuvieron los códigos definitivos (ver figuras 1 y 3). Se consideró la frecuencia en las preguntas de opción múltiple y la cantidad de menciones de las categorías definidas en las abiertas, en este caso se analizó la jerarquía, es decir, los códigos con mayores o menores menciones, cantidad de referencias (citas) en las respuestas vertidas por los profesores.

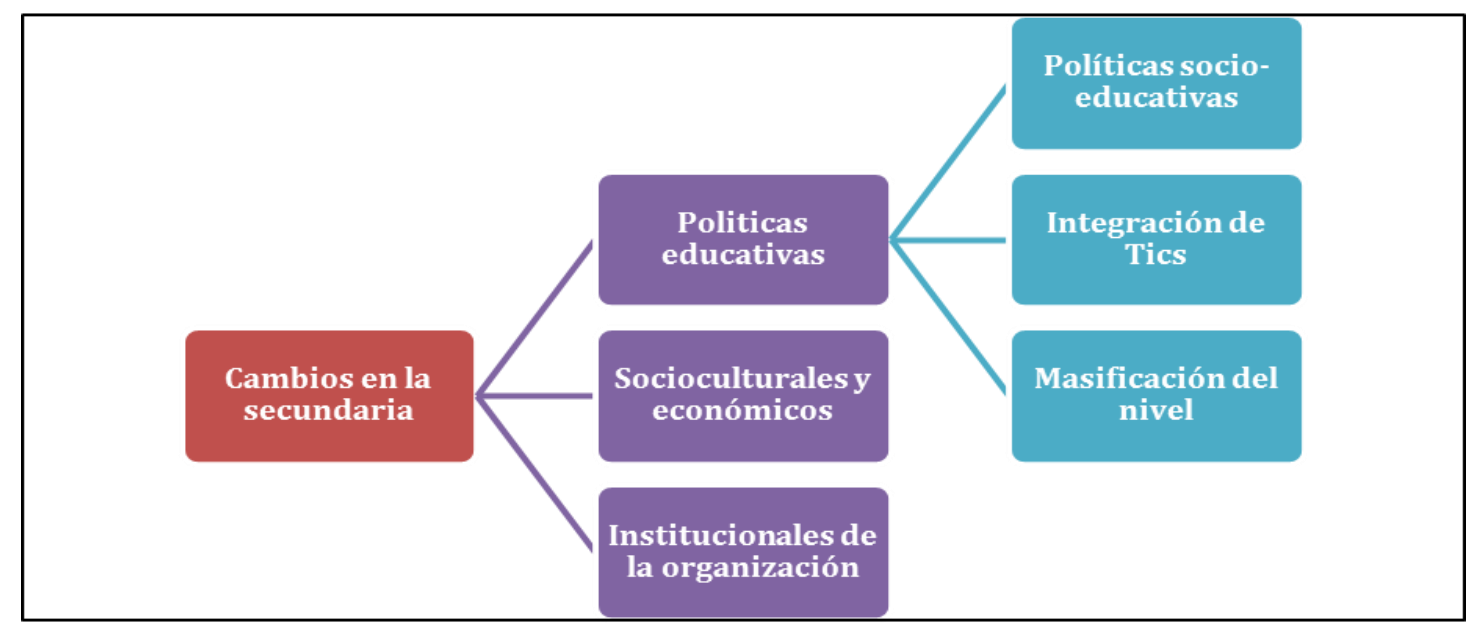

Figura 1. Categorías pregunta abierta: Transformaciones del nivel secundario 


\section{La transformación de la escuela secundaria}

Al indagar sobre la transformación de la escuela y los cambios ocurridos en la tarea de los profesores, casi la totalidad (94\%) de los entrevistados coincide en que su trabajo se ha alterado profundamente, ya sea que lo considere "muy transformado" o "más o menos transformado" (ver figura 2).

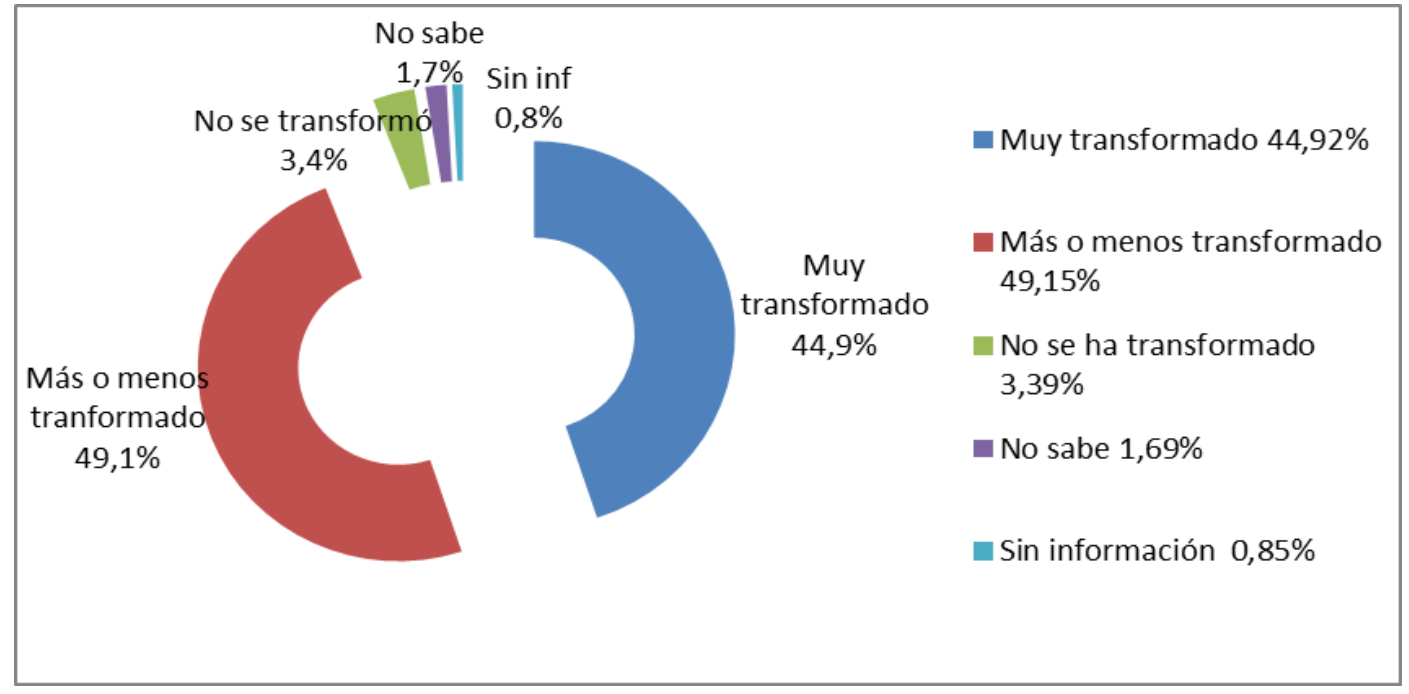

Fuente: Elaboración propia.

Figura 2. Transformaciones del trabajo docente durante los últimos años. Porcentajes

Estos resultados pueden ser interpretados, no sólo en relación con los recientes cambios experimentados desde la nueva Ley de Nacional de Educación de 2006, sino en función de la antigüedad y trayectoria previa de los profesores. Se trata de una población docente con muchos años de experiencia, por lo que a las reformas actuales se suman las vividas en los años noventa. Ambos procesos, para la mayoría, implica cierta desestabilización respecto de la formación docente recibida, por lo que precisan revisar las características conocidas del adolescente y el propio rol.

Actualmente, los lineamientos y políticas para este nivel educativo destacan a los alumnos como sujetos de derecho y la necesidad de garantizar la trayectoria escolar de todos los que ingresan a secundaria, aumentando la retención. Para ello se propone diversificar los modos de evaluación, acreditación y promoción de los alumnos; alterar los formatos, tiempos y agrupamientos de la gramática escolar consolidada. Estos acuerdos establecen "una nueva escuela secundaria", es decir, más inclusiva, democrática y equitativa, capaz de garantizar la trayectoria escolar exitosa de los adolescentes. Por el contrario, el formato anterior se ha descrito en términos de un modelo de "selección por exclusión" que expulsaba a los estudiantes que no se adaptaban a los requerimientos de la cultura escolar y convertía el fracaso en un asunto individual (Ziegler, 2011).

Como se ha señalado por otros estudios (Bolívar, Fernández y Molina, 2004; Ávalos y Sotomayor, 2012) la cuestión de la identidad docente constituye un problema complejo; se refiere a la forma en que los docentes definen, perciben y asumen sus tareas y al modo en que entienden sus relaciones con otros actores. La trayectoria profesional atraviesa importantes procesos de revisión y cambio (Vezub, 2011) debido a la masificación de la secundaria y a las nuevas exigencias docentes que generan los cambios mencionados en las políticas.

Para Dubar (2002), las formas de identificación en los diversos campos -incluso los profesionalesse encuentran en movimiento; desde formas de identificación comunitarias hacia otras 
asociaciones, más efímeras y provisionales que dan primacía a los sujetos por encima de los grupos de pertenencia, la identidad heredada. El autor define la "crisis de las identidades" como perturbaciones, desequilibrios en las relaciones relativamente consolidadas, estabilizadas en los procesos de categorización de uno mismo y de los demás, que alteran las formas anteriores de constitución de la subjetividad. Por su parte, Grimson (2011) analiza la crisis cultural en relación con la noción de identidad, al considerarla como una suspensión del sentido común y del imaginario acerca de quiénes somos.

Las transformaciones percibidas en los últimos años por los docentes (inclusión, permanencia de estudiantes diversos, desatentos, con códigos y usos del lenguaje singulares, que no poseen el hábito escolar esperado y frente a los cuales deben pensarse otras manera de "dar clase"), ponen de manifiesto la crisis de las identidades y la búsqueda de nuevas formas de identificación. Se observan dificultades en los profesores para establecer normas, hallar el significado de su trabajo y de la experiencia escolar de los alumnos. Su tarea por momentos les resulta agobiante, al sentirse solos, desprovistos de la autoridad, legitimidad y solidez que antes les otorgaba el programa institucional de la modernidad (Dubet, 2006).

Entre los cambios ocurridos, el proyecto focalizó en la integración de las TIC en la enseñanza, preguntando a los profesores acerca de su potencial para motivar a los alumnos y favorecer el aprendizaje (ver tabla I). La mayoría (82\%) manifestó al respecto una actitud y opinión positiva.

Tabla I. Incidencia de la integración de las TIc en el aprendizaje y motivación de los alumnos

\begin{tabular}{lcc}
\hline $\begin{array}{l}\text { ¿Las TIc favorecen el aprendizaje } \\
\text { y la motivación? }\end{array}$ & Cantidad & Porcentaje \\
\hline Muy positiva y significativamente & 38 & 32.20 \\
Positiva y significativamente & 59 & 50.00 \\
Favorece muy poco & 18 & 15.25 \\
No favorece, entorpece & 1 & 0.85 \\
No sabe & 2 & 1.69 \\
Total & 118 & 100 \\
\hline
\end{tabular}

Fuente: Elaboración propia.

Sólo un 15\% respondió que las TIC "Favorecen muy poco" y menos del 1\% consideró que son un obstáculo para la tarea de enseñanza y el aprendizaje. Los datos obtenidos coinciden con otros estudios que señalan cómo más allá de la empatía tecnológica de los docentes y de las discusiones respecto de su carácter de "inmigrantes digitales", las actitudes docentes hacia las tecnologías comenzaron a transitar la senda de la aceptación (Dussel, 2011). ${ }^{7}$ La mayoría las utiliza en su vida personal y con fines profesionales, frecuentemente para buscar información, comunicarse, capacitarse y, gradualmente, aumenta el número de los que comienzan a incorporarlas con fines pedagógicos en el aula. Estos cambios son en parte resultado de las políticas educativas emprendidas y de los programas de formación continua que las fomentan, junto con la omnipresencia de los dispositivos tecnológicos en la vida cotidiana y en las instituciones educativas.

La importancia dada a las TIC entre las transformaciones recientes que atraviesa la escuela secundaria es mencionada espontáneamente por los profesores del estudio. Al consultarles cuáles son los cambios que tienen mayor incidencia en la transformación de las instituciones y de

\footnotetext{
${ }^{7}$ Los datos de la encuesta encargada por la Unidad de Planteamiento de la Educación del Ministerio de Educación de la Argentina y efectuada por la sede del IIPE UNESCo Buenos Aires a finales de 2010, sobre una muestra nacional que incluyó a docentes de diversos niveles y regiones del país (Dussel, 2011), indican que el $63 \%$ de los docentes cree que las nuevas tecnologías tienen un impacto positivo en la motivación de los estudiantes, aunque la confianza en su capacidad para fomentar la creatividad y el aprendizaje decrece (46\%), al igual que para fomentar la crítica ante el conocimiento y la información (37\%).
} 
su tarea docente, las tecnologías y, específicamente, el "Programa Conectar Igualdad", están entre los más nombrados (como se verá en el siguiente apartado). Los docentes refieren a la distribución reciente de notebooks a los estudiantes, a la integración de tic y la facilitación del aprendizaje ocurrida con la incorporación de las computadoras en el aula entre los principales cambios.

Si bien las tecnologías han creado brechas y tensiones entre las concepciones clásicas de lo escolar sostenidas por la cultura profesional docente y la cultura digital de los alumnos; también brindan una oportunidad para romper con los clásicos formatos escolares y repensar la transmisión, los vínculos con el saber, el sentido de la actividad escolar (Brito, 2012; Levy, 2013). Estos nuevos modos de vincularse y acceder al conocimiento conducen a que profesores y alumnos cuestionen los lugares que cada uno ocupa en el aula.

\section{La percepción de los cambios desde la perspectiva de los profesores}

Los cambios mencionados por los profesores se agruparon en tres dimensiones: políticas educativas (125 menciones), modificaciones socio-culturales y económicas (40 menciones), y cuestiones organizacionales e institucionales (21 menciones).

Las mayores transformaciones se perciben en las políticas educativas, más específicamente se nombran: los cambios en las políticas sociales educativas (47 citas/menciones), la entrega de las notebook a los alumnos (41 citas) y, la masificación, obligatoriedad de la secundaria (37 citas). Las dos últimas medidas y estrategias se vinculan con la democratización de la educación, la incorporación de nuevos estudiantes y provocan el replanteo de la profesión docente. El tercer cambio, en orden de importancia, que los profesores identifican en las políticas educativas, son las modificaciones de los diseños curriculares. Luego, mencionan otras medidas de gobierno como la Asignación Universal por Hijo y otros planes de ayuda/mejora social, las nuevas pautas de acreditación y evaluación de los estudiantes del nivel y, de manera general los cambios en las leyes educativas de la nación (Ley 26026, de 2006) y de la provincia de Buenos Aires (Ley 13688, de 2007).

Para algunos profesores estas políticas provocaron un cambio de mirada respecto de los estudiantes, de su labor docente y de la calidad educativa. En estos casos entienden que las modificaciones curriculares, por ejemplo, presentan nuevos enfoques pedagógicos, formas de enseñanza y fundamentos vinculados a los principios de inclusión, democratización y equidad educativas. Las políticas de asignación universal por hijo para familias sin empleo formal, la obligatoriedad y masificación del nivel conllevan de este modo, una nueva pedagogía y sujeto de derecho; sin embargo no todos los docentes se adhieren a esta perspectiva:

La Ley de educación nacional y provincial que promulgó la inclusión, por la cual se decide trabajar desde la calidad educativa, dejando la concepción elitista de la antigua secundaria $(2: 144)$

Creo importante la lectura de la Ley Nacional y Provincial de Educación, así como también los Diseños curriculares prescriptivos y demás normativas vigentes donde apuntan a los alumnos como sujetos de derecho (6:17)

Creo que uno de los temas principales es el asistencialismo, la escuela se ha transformado en un medio de asistencia. La mayoría de los estudiantes pretenden que todo debe ser dado por el solo hecho de asistir al aula, no hay compromiso (6:45)

La escuela se ha transformado en el lugar de resolución de problemas domésticos, vecinales y sociales. De permanencia, por los planes sociales o becas (2:262)

Como se aprecia en los fragmentos anteriores, las reformas y cambios vigentes son valorados de 
manera positiva en algunos casos, y negativa en otros, al amenazar el orden y el formato escolar tradicional. Algunos profesores critican las políticas implementadas por considerarlas "apuradas" y "descontextualizadas", realizadas sin la participación y consulta a los docentes y la debida planificación. De este modo, coexisten concepciones profesionales más conservadoras y elitistas, al pensar que la masificación de la escuela secundaria ocasiona la pérdida de su nivel y calidad al disminuir la exigencia y transformarse en un espacio de contención social, donde el docente "se ve obligado a dejar de lado lo pedagógico" (6:1); con otras más progresistas.

En esta muestra de profesores, parece ganar terreno, paulatinamente, la aceptación del cambio en términos de una escuela más democrática e inclusiva. Sin embargo, esto no significa que la tarea de los educadores se viva sin tensiones, por el contrario, provoca perplejidad e incertidumbre, como manifiesta uno de los entrevistados:

Uno de los principales cambios es la inclusión y no saber qué hacer con la heterogeneidad de los jóvenes en el aula (2:89)

Entre las políticas educativas que más mencionan los profesores (41 citas), se encuentra la implementación del Plan Conectar Igualdad:

La integración de nuevas competencias (tecnologías, comunicacionales, pedagógicas) $(2: 127)$

Incorporación de las máquinas (netbooks) que han facilitado los aprendizajes de los alumnos $(2: 137)$

Para los docentes, las computadoras en el aula permiten motivar a los estudiantes en su aprendizaje. Esto muestra un viraje en sus opiniones y actitudes hacia las tecnologías, ya que durante mucho tiempo los docentes aludían a la brecha digital como un obstáculo para generar experiencias de aprendizaje y de interacción cultural igualadoras (Southwell, 2013).

No obstante, los mismos profesores que subrayan el potencial de las TIC para motivar el aprendizaje, señalan también errores en la implementación del Programa. En primer lugar, se critica la lenta entrega de los equipos, hay docentes y estudiantes que no cuentan con las computadoras para trabajar, ya sea por trabas administrativas o porque no hay suficientes referentes tecnológicos en las instituciones que brinden el apoyo necesario, entre otros factores. En segundo lugar, la falta de capacitación para acompañar el uso del recurso material. Si bien entienden que la incorporación de tecnología es importante, todavía su apropiación y uso efectivo en las instituciones, no está plenamente garantizado, al igual que lo señalado en otros países e investigaciones (Garrison y Anderson, 2005; Sancho, 2010).

Los docentes mencionan, además -en términos generalmente negativos- los cambios socioculturales y económicos. En este caso se refirieren a los problemas de adicciones de los estudiantes, la violencia escolar, la crisis económica de las familias y de los docentes, la pérdida de valores, las transformaciones en las culturas y códigos adolescentes y juveniles. Los actores de la escena educativa son caracterizados a partir de "la situación de vulnerabilidad de la escuela pública, del docente trabajador, de las familias y de los alumnos" (1:134).

La familia es el actor social "perjudicado" por estas transformaciones. Los docentes opinan que se ha producido una digresión, ruptura de los lazos afectivos, intrafamiliares debido a la crisis económica que atraviesan muchas familias. Todo ello afecta el trabajo de las escuelas y la posibilidad de enseñar:

\footnotetext{
${ }^{8}$ Hay que mencionar que el mismo año en que se recogieron los datos de la investigación fue aprobada la Especialización docente de Nivel Superior en Educación y tıc que comenzó en 2013 y fue ampliando la oferta a los docentes de los distintos niveles y modalidades educativas del país (Res. Ministerial N856/2012).
} 
Hay muchos problemas sociales que debe sortear el docente en su tarea cotidiana, y dejar de lado lo pedagógico (5:5)

Hay una mutua sospecha y desconfianza, los profesores no confían en las familias y las familias no confían en los docentes. En el mismo sentido, los datos de otras investigaciones (Alliaud y Vezub, 2012) muestran que para los docentes su trabajo no es reconocido ni valorado por la familias; mientras que los docentes acusan a éstas por la falta de acompañamiento en el aprendizaje de sus hijos y la actitud despreocupada por el acontecer escolar que se evidencia en la poca participación que tienen cuando son convocados (Tenti, 2006).

En tercer lugar, los profesores consultados se refieren a las cuestiones organizacionales e institucionales que han alterado a la escuela secundaria y su trabajo. Por ejemplo, los cambios en los reglamentos de convivencia de las instituciones, las modificaciones administrativas, las normas referidas a la promoción y los nuevos diseños curriculares para el nivel. Con mayor énfasis reiteran las transformaciones en la acreditación y evaluación de los alumnos, vinculadas además con la calidad educativa.

Cambios constantes y continuos en los currículos, en los contenidos, en la organización y administración de la escuela secundaria (6:31)

Modificaciones administrativas en la cantidad de mesas de examen, en las posibilidades otorgadas a los alumnos para rendir, en los criterios de evaluación, en los períodos de orientación y evaluación (6:32)

Reducción del nivel de exigencia y de criterios de evaluación (6:46)

Para los docentes, los cambios en el régimen de evaluación son una forma de reducir el nivel de exigencia académica que conduce a disminuir la calidad educativa. Por ello se perciben como transformaciones negativas y amenazantes para la identidad profesional, ya que impiden la tarea de enseñanza y la comprobación del conocimiento trasmitido. Las transformaciones en la organización escolar equivalen a una escuela más flexible, "fácil y relajada", no son formas de contemplar las diversas trayectorias de los estudiantes que garanticen la inclusión y el derecho a la educación, como establecen los nuevos lineamientos y regulaciones para este nivel.

El logro de la inclusión implica complejos desafíos, tanto para la organización institucional como para las identidades y culturas profesionales docentes. En relación con lo primero es necesario repensar las lógicas y dinámicas institucionales: las normas, el régimen académico, el uso de tiempos y espacios para la enseñanza y el aprendizaje, ampliando la concepción de escolarización vigente. Aspectos que han sido encuadrados dentro del denominado núcleo duro e inamovible de la escuela secundaria argentina (Tiramonti, 2011).

\section{Viejos y nuevos problemas de la escuela secundaria}

Para el análisis de los problemas de la escuela secundaria señalados por los profesores, se construyó un sistema de doble categorización (ver figura 3). Por un lado se clasificaron los asuntos, los elementos o aspectos del nivel sobre los cuales se identificaron las principales dificultades. Por otro lado, se prestó atención a los actores mencionados y a sus problemas, con lo cual se elaboró un segundo grupo de categorías. 


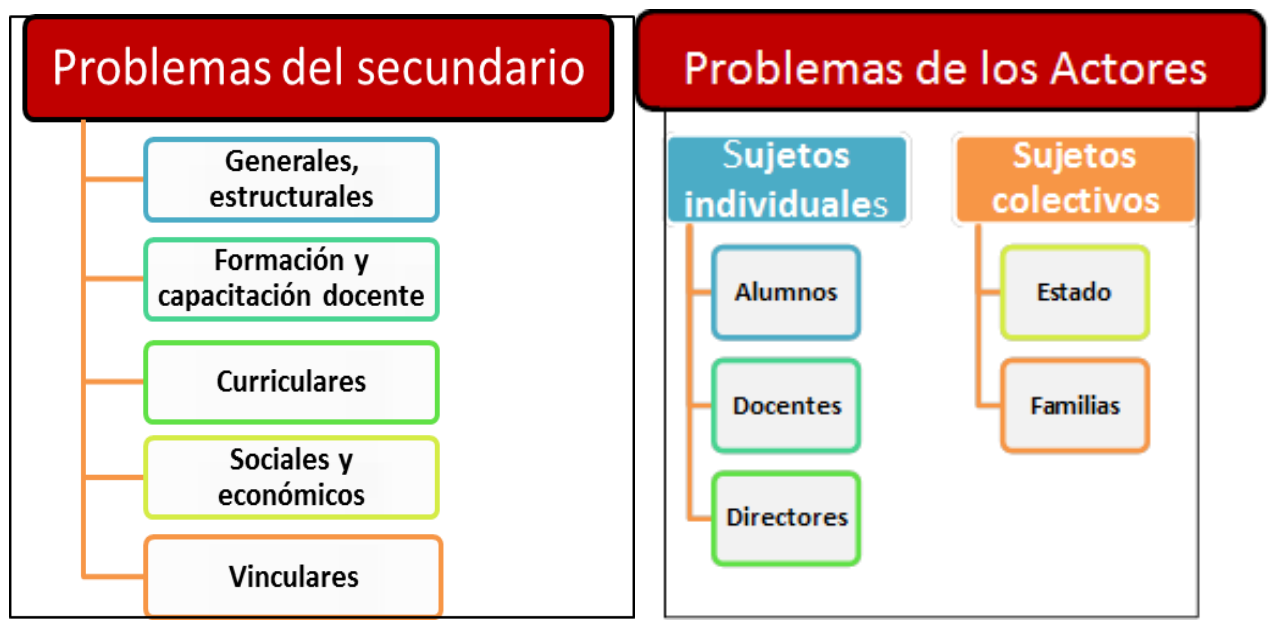

Figura 3. Doble categorización: Problemas más graves que enfrenta el nivel secundario

En el análisis realizado a partir de los actores, aparecen diversos sujetos involucrados en las problemáticas que los profesores identifican: el Estado, el gobierno/autoridades, los directores, los docentes, las familias, los alumnos. Los problemas de los sujetos individuales (docentes, alumnos, directivos) ocupan un lugar preponderante con 159 citas o menciones. A éstas se agregan otras 25 respuestas que aluden al sujeto colectivo familia, que no acompaña o acompaña poco a sus hijos, no se comprometen con su educación, etc. Por último, 38 citas mencionan al Estado y se identifican problemas como la falta de inversión, de presupuesto, la mala infraestructura y el abandono.

Al focalizar en los problemas de los actores individuales, se observa que las cuestiones que más preocupan son: el ausentismo de estudiantes y de docentes, la falta de interés de los estudiantes y de compromiso de los docentes y directores. Esto provoca el desgano y desmotivación de los principales protagonistas de la escena educativa para enfrentar la tarea que los convoca, configurando lo que hemos caracterizado como "trayectorias profesionales cerradas, rígidas y en declive" frente al nuevo panorama de la escolarización contemporánea (Vezub, 2011).

Uno de los resultados a destacar es la paridad de respuestas encontradas en los problemas que tienen a los "alumnos" y a los "docentes" como protagonistas (68 y 60 menciones, respectivamente). Esto evidencia un reposicionamiento de los profesores respecto de su tarea y de los problemas de la escuela, a la vez que muestra desplazamientos en las identidades profesionales previamente constituidas. Nos referimos a desplazamientos porque tradicionalmente los profesores pensaban que el fracaso escolar y los bajos resultados académicos de los estudiantes eran un problema, cuya responsabilidad principal recaía en los alumnos y en sus familias, o en todo caso al contexto socioeconómico y las condiciones de vida. Por el contrario, y más allá de cómo perciben las transformaciones (de manera positiva, negativa, como amenaza al orden establecido o, como parte de la democratización de la educación); los docentes han comenzado a ubicarse como sujetos de la acción y protagonizan los problemas. Es decir que no sólo los "padecen", sino que ellos mismos forman parte de las dificultades que atraviesa el nivel secundario en el país. Entre los problemas de los docentes se encuentran, como ya se ha señalado, el ausentismo como manifestación evidente y, de manera más subjetiva, el desánimo y la desmotivación:

El malestar docente y su pesimismo (1:76)

Nivel de desaliento de los docentes por múltiples factores. Sensación de desánimo, de frustración, de vulnerabilidad (1:5)

Falta de adaptación de los docentes a los cambios (1:44) 
Están esperando estudiantes que ya no están (4:79)

Falta de vocación (1:78)

Ausentismo docente $(1: 150 ; 179 ; 191 ; 126 \ldots)$

La falta de compromiso y desinterés se reiteran en varias respuestas y se puede asociar con el ausentismo docente. Consideramos que constituye una de las facetas visibles de la denominada "crisis de la identidad profesional docente". En algunos casos los profesores hablan de la resistencia de sus colegas, las dificultades que tienen para aceptar los cambios de la escuela secundaria, conocer y acercarse a la realidad sociocultural de los estudiantes y resignificar su rol al "observar a los adolescentes como alumnos problemáticos" (4:34).

Considerando el otro sistema de categorías, los problemas del secundario que identifican los profesores, la mayoría de las respuestas refieren a dificultades generales y estructurales (71 menciones), problemas históricos, de larga data en el nivel. Por ejemplo: la falta de inversión, infraestructura, mantenimiento de edificios y equipamiento de las escuelas, aulas con exceso de estudiantes, docentes sobrecargados de funciones, la deserción y la repitencia, el currículo descontextualizado y desarticulado con el mundo del trabajo, el modelo escolar exclusor y elitista de la secundaria.

Hay en este grupo de problemas numerosas menciones al déficit de infraestructura, recursos didácticos, mantenimiento e higiene de las escuelas que, según los profesores, no acompaña la proclama, principios y objetivos de las leyes educativas en términos de inclusión. Entre los problemas históricos se encuentran, además, la repetición de cursos y la deserción; también aunque en menor medida- se habla de la escasa conexión de la escuela y del currículum secundario con el mundo del trabajo y del predominio de la enseñanza tradicional. Algunos profesores consideran que la "gramática de la escuela secundaria" es un obstáculo. Es probable que la utilización de este concepto y su identificación como problema sea consecuencia de los procesos, discursos, materiales y contenidos que circulan en la formación en servicio y que se vienen trabajando en diversas jornadas institucionales. Varios docentes caracterizan a la escuela como:

Solapadamente exclusora (1:59)

Impide trayectorias escolares diferentes (1:66)

Hay una gramática escolar que no se cuestiona ni se modifica (1:81)

Los formatos están desactualizados (1:157)

En segundo lugar, se destacan los problemas referidos a la dimensión de la formación y actualización profesional de los docentes. En este caso se encontraron 55 respuestas que se analizan en el próximo apartado. En tercer lugar, los profesores ubican los problemas de la dimensión curricular-pedagógica (43 citas) al aludir a la distancia entre los contenidos prescriptos por los diseños curriculares y la realidad de los alumnos, consecuencia de la enseñanza atomizada, tradicional e enciclopedista del conocimiento escolar. Esto lleva a una situación de "falta de... o escaso aprendizaje", donde las dificultades de comprensión, la carencia de habilidades de lectura y expresión de los estudiantes ocupan el primer plano.

Los chicos no le encuentran ni sentido ni utilidad a lo que enseñamos (1:19)

Modelo disciplinar fragmentado (1:184)

La brecha de interés de los estudiantes y la propuesta docente (1:114) 
Dificultades de integrar contenidos con problemáticas y enfoques basados en la realidad actual (1:32)

Ausencia de proyectos interdisciplinares, transversales y multidisciplinares (4:85)

Es menor la referencia a cómo estas cuestiones se vinculan con las propias estrategias pedagógicas de los profesores y con las dificultades para modificar las prácticas de enseñanza. No obstante, unos pocos mencionan la falta de habilidades didácticas y de apropiación de los nuevos diseños curriculares. En este caso hablan en tercera persona, acerca de otros colegas empeñados en seguir aplicando formas tradicionales de enseñanza, sin adaptarse a la heterogeneidad de los alumnos.

Mala implementación de los contenidos de los diseños por parte de los docentes (1:83)

Falta de actividades creativas (1:120)

En cuarto lugar aparecen, con similar cantidad de referencias, los problemas de la sociedad en general (21 citas) y las dificultades vinculares (17 menciones). En la primera los docentes se refieren a la violencia y agresividad de la sociedad, las adicciones, las transformaciones socioculturales, la desigualdad socioeconómica y la situación de alumnos que trabajan, además de estudiar o deben cuidar a sus hermanos menores.

A pesar de que los cambios intergeneracionales en los vínculos y en la autoridad son temas sobre los que los expertos insisten (Martuccelli, 2009; Skliar, 2012), los docentes de nuestro estudio todavía no los visualizan como algo central. Las dificultades de los profesores para vincularse o la poca tolerancia con símbolos, prácticas y consumos culturales de los adolescentes, no aparecen entre los problemas principales. Varios de quienes las mencionan, le atribuyen un sentido negativo, al hablar de la pérdida de la autoridad del profesor, o en clave moral, de la "falta de respeto" de los estudiantes. Entre las respuestas categorizadas en la dimensión vincular algunas aluden a la violencia entre pares y hacia los docentes.

Los especialistas coinciden al señalar que la universalización del nivel requiere de una modificación profunda de la naturaleza y matriz fundante en base a la cual se desarrolló. Es por ello que frente a las dificultades de la expansión de la escolarización secundaria en el país, en tanto ésta ha seguido un proceso conceptualizado en términos de "incorporación/expulsión simultánea" (Felicitas, 2012), o "selección por exclusión" (Ziegler, 2011), varias investigaciones (Tiramonti, 2011; UNICEF-UnGS, 2012; Terigi, Briscioli, Scavino, Morrone y Toscano, 2013) han estudiado experiencias institucionales alternativas, que modifican elementos del modelo organizacional para generar mejores condiciones, aumentar la retención y graduación de los jóvenes. ${ }^{9}$ Dichas investigaciones identifican que el vínculo pedagógico es una variable clave para sostener las trayectorias educativas de los adolescentes (Nobile, 2011). La relación de confianza, ayuda, mayor cercanía, trato personal entre profesores y estudiantes -a diferencia de lo que sucede en los formatos escolares tradicionales- genera un clima escolar positivo y favorece el aprendizaje. Sin embargo, para los profesores de nuestro estudio, los aspectos vinculares no constituyen todavía un problema central. Posiblemente este rasgo remite al núcleo duro y fundante de la identidad del profesor de secundaria, basada en la transmisión del contenido, sin demasiado lazo afectivo.

\footnotetext{
${ }^{9}$ Se trata de diversos programas y políticas alternativas, tanto nacionales como de los gobiernos provinciales y la Ciudad Autónoma de Buenos Aires, que buscan estrategias de reingreso al nivel una vez abandonado, o estrategias puente o directamente lograr la terminación y egreso de los estudios medios. Por ejemplo: Escuelas de Reingreso de la Ciudad de Buenos Aires, Plan FiNes del Ministerio de Educación Nacional, el Programa de Inclusión y Terminalidad de la Escuela Secundaria de la provincia de Córdoba; Centros de Escolarización Secundaria para Jóvenes de la Provincia de Buenos Aires.
} 


\section{Expectativas de formación y desafíos de las políticas}

El otro tópico que indagó la investigación fue si en el marco de las trasformaciones de la escuela secundaria, los docentes habían realizado actividades de formación en servicio y de qué manera las valoraban.

Casi todos los profesores encuestados (91.5\%) recibieron capacitación en los últimos cinco años. No obstante, los porcentajes descienden al consultarlos sobre la valoración y el aporte de esta formación para su trabajo. La mitad considera que fue útil y relevante, mientras que un tercio (36\%) relativiza su contribución al evaluarla como "más o menos útil y relevante". Estos datos se complementan con los obtenidos en la pregunta abierta referida a los problemas más graves que enfrenta la secundaria, ya que la formación de los profesores es mencionada en segundo lugar de importancia.

El segundo de los problema para los profesores consultados, radica en:

La falta de capacitación docente (1:21)

El bajo nivel académico docente (1:79)

La formación de los docentes (1:115 - 156 - 162 - $183-210$-2:8)

Algunos profesores van más allá, al manifestar su preocupación por la ausencia de espacios de reflexión sobre la práctica del aula y consideran necesaria mayor formación e intercambio entre pares:

Muy pocos espacios de reflexión (2:52)

La falta de espacios para evaluar la propia tarea (1:132)

Estos hallazgos manifiestan la necesidad de revisar las propuestas, las políticas y los programas de formación en servicio para acercarlas a los problemas de la práctica y a las necesidades formativas de los colectivos docentes, recuperando las inquietudes de las escuelas y construyendo nuevas estrategias de trabajo. En las respuestas se recoge una demanda explícita al Estado y a las autoridades educativas sobre la capacitación. En este sentido, las políticas de formación en servicio, entre ellas el actual Programa Nacional de Formación Permanente (en curso desde 2014) por su envergadura, tienen la oportunidad, responsabilidad y el desafío de responder a dichas expectativas de formación y desarrollo de los profesores.

Por último, al analizar el tipo de programa de formación en el cual participaron los profesores, prevalece el formato curso como dispositivo dominante. La mayoría (82\%) asistió a cursos de distintas temáticas (ver tabla II) entre las que predominan las didácticas específicas. En segundo lugar, se evidencia un crecimiento de trayectos de mayor duración: los ciclos de licenciaturas, postítulos y especializaciones que buscan profundizar en un área o campo educativo particular, de manera sistemática. 
Tabla II: Profesores que realizaron capacitación docente según áreas temáticas

\begin{tabular}{l|c|c}
\hline Contenido de la formación continua/área temática & Cantidad & Porcentaje \\
\hline Didácticas específicas; contenidos de las disciplinas & 67 & 40.8 \\
\hline TICs, tecnologías digitales, nuevos entornos de aprendizaje & 39 & 23.8 \\
\hline $\begin{array}{l}\text { Otros temas pedagógicos (por ej. didáctica general, } \\
\text { evaluación, problemas de aprendizaje, currìculum). }\end{array}$ & 19 & 12.8 \\
\hline $\begin{array}{l}\text { Planificación y gestión educativa } \\
\text { Temas transversales y contemporáneos: sujetos y problemas } \\
\text { socioculturales de la educación }\end{array}$ & 11 & 11.6 \\
\hline Análisis de las prácticas pedagógicas & 21 & 6.7 \\
\hline Total* & 164 & 100 \\
\hline
\end{tabular}

(*) El total no es igual a la cantidad de docentes porque hay docentes que realizaron más de un curso. Fuente: elaboración propia.

Un 12\% de los profesores se encuentran realizando o, han finalizado, este tipo de ofertas, en las universidades o a través de los postítulos ofertados desde el Ministerio de Educación. Tercero, un $5 \%$ de los profesores participaron de capacitaciones para obtener un ascenso en la carrera docente y aspirar a cargos directivos de las instituciones educativas o de supervisión del nivel.

Si bien los profesores destacan entre los problemas de los sujetos docentes el desánimo y la falta de compromiso, a esto sigue el reconocimiento de la importancia que tiene la formación, para lograr una mejor enseñanza y escolarización de los adolescentes. Ambas problemáticas se dirigen a repensar la subjetividad y el posicionamiento de los actores en la escena educativa, es decir, la necesidad de revisar las identidades profesionales.

\section{Conclusiones}

En relación con la pregunta inicial de la investigación: ¿Cómo se interpela la trayectoria e identidad de los profesores en el contexto de la masificación de la escuela secundaria y del nuevo modelo inclusivo? Los datos recogidos evidencian un profesorado consciente de los problemas, que ubica a su formación y motivación como parte de las dificultades. La conciencia en torno a la capacitación como dimensión clave y estrategia de cambio, si bien es alentadora, obliga a intensificar los compromisos de las políticas con el desarrollo profesional docente, a elevar la calidad y pertinencia de las propuestas, mejorando la articulación entre el diseño de los programas y su implementación.

Si la identidad profesional docente es un proceso de construcción de sentido respecto del trabajo de enseñar que abarca tanto motivaciones, expectativas, personales como concepciones referidas a la tarea y a la función de la escuela que se consolidan durante la formación inicial y modifican luego, en la trayectoria posterior; no es posible soslayar la relevancia de los espacios de formación continua en su configuración y cambio.

Entre los resultados se destacan diversas ideologías profesionales y apreciaciones sobre las transformaciones experimentadas, lo que muestra diferentes bases identitarias en los docentes. Algunas de estas identidades en crisis se caracterizan por instituir la tarea docente bajo un orden democrático e igualador (apropiándose del discurso de la actual política educativa). Mientras que otras se constituyen en torno a un discurso conservador que defiende el statu quo de la docencia y de la escuela, dejando poco lugar para la inclusión educativa del nivel y replantear la gramática y la cultura institucional tradicional del secundario.

A modo de hipótesis parecen delinearse tres identidades docentes. Las primeras, de rasgos progresistas, aceptan el cambio de época en la educación, las nuevas políticas para enseñar a nuevos sujetos. Las segundas son identidades defensivas, en repliegue y rígidas (Dussel, 2007; Vezub, 2011; Brito 2012) que manifiestan nostalgia respecto a otros tiempos y maneras de 
ejercer la docencia, sin disponerse a modificar los rasgos básicos de la profesión. Aunque estos profesores saben de antemano que sus modos ya no son eficaces, que existe un anacronismo entre sus prácticas, sus expectativas y las de los estudiantes, no están dispuestos -o no puedenrenunciar a ellas. Hacerlo significa dejar de ser lo que son, desaprender, caer en la ambigüedad y la incertidumbre, abandonar la profesión, el trabajo para el cual se formaron. En un lugar intermedio, encontramos en tercer lugar, identidades estables: docentes que aceptan algunos cambios y se replantean cómo hacer para mejorar el interés de los estudiantes en las clases. Por ejemplo, incorporando tecnologías, imágenes, haciendo que los estudiantes trabajen en grupos, etc. pero, sin renunciar a las evaluaciones individuales, las mismas y al mismo tiempo para todos, al programa único, a la enseñanza simultánea.

Las identidades progresistas son encarnadas por profesores que sienten que se ha agotado el formato escolar y las estrategias con las que llevan a cabo la enseñanza. Intentan afrontar la construcción y búsqueda de una nueva identidad profesional más acorde con los escenarios escolares actuales.

Koselleck (1993) aporta dos conceptos que recuperamos para pensar la construcción y la dinámica de las identidades y de las trayectorias profesionales docentes: el espacio de la experiencia y el horizonte de expectativa. La crisis o conflicto se produce, según el autor, porque cada vez aumenta más la diferencia entre estos dos ámbitos que entrecruzan pasado y futuro. Por ello ocurre que en los espacios de formación continua y de reflexión sobre la tarea, en las instituciones, ya sea de manera solitaria o colectiva, las vivencias, las experiencias atravesadas en el desempeño real de los profesores (el espacio de la experiencia) son puestas en tensión con aquello que se desea, con lo que se espera de la profesión, lo que se ansía (el horizonte de expectativa). En esta puja, por ahora, parecería que los profesores sienten que su horizonte de expectativa lleva las de perder. Quizá, como insinúa Koselleck, es necesario romper el horizonte de expectativa para fundar una nueva experiencia.

Es en este entrecruzamiento, donde probablemente puedan configurarse nuevas identidades docentes, más acordes a los nuevos sujetos de la educación, a las tareas y funciones que la escuela secundaria requiere. Sin que ello signifique la renuncia o el abandono de algunos núcleos básicos de la profesión: la enseñanza de contenidos relevantes, la transmisión cultural, la formación de ciudadanos críticos. El desafío es cómo se reactualizan dichos núcleos y cómo se viabilizan en los nuevos escenarios escolares y sociales.

\section{Referencias}

Alliaud, A. y Antelo, E. (2008). El fracaso de enseñar. Ideas para pensar la enseñanza y la formación de los futuros docentes. En D. Braislovsky (Coord.), Sentidos perdidos de la experiencia escolar (pp. 27-56). Buenos Aires: Noveduc.

Alliaud, A. y Vezub, L. (2012). El oficio de enseñar: sobre el quehacer, el saber y el sentir de los docentes argentinos. Revista Diálogo Educacional, 12(37), 927-952. Recuperado de http://www2.pucpr.br/reol/pb/index.php/dialogo?dd1=7211\&dd99=view\&dd98=pb

Ávalos, B. y Sotomayor, C. (2012). Cómo ven su identidad los docentes chilenos. Perspectiva Educacional, 51(1), 77-95. Recuperado de http://www.perspectivaeducacional.cl/index.php/peducacional/article/view/74

Bolívar, A., Fernández, M. y Molina, E. (2004). Investigar la identidad profesional del profesorado: una triangulación secuencial. Forum Qualitative Research, 6(1). Recuperado de http://www.qualitative-research.net/index.php/Fas/article/view/516 
Bolívar, A., Gallego, M. J., León, M. J. y Pérez, P. (2005). Políticas educativas de reforma e identidades profesionales: el caso de la Educación Secundaria en España. Archivos Analíticos de Políticas Educativas, 13(45). Recuperado de http://epaa.asu.edu/ojs/article/download/150/276

Brito, A. (2012). Reinventar la escuela: límites y posibilidades desde la perspectiva de los profesores. En M. Soutthwell (Comp.), Entre generaciones. Exploraciones sobre educación, cultura e instituciones (pp. 133-144). Rosario: Flacso-HomoSapiens.

Corea, C. (2005). Pedagogía y comunicación en la era del aburrimiento. En C. Corea e I. Lewcowicz (Eds.), Pedagogía del aburrido. Escuelas destituidas, familias perplejas. Buenos Aires: Paidós.

Dubar, C. (2002). La crisis de las identidades. La interpretación de una mutación. Barcelona: Belaterra.

Dubet, F. (2006). El declive de la institución. Profesiones, sujetos e individuos en la modernidad. Barcelona: Gedisa.

Dussel, I. (2007). Más allá de la crisis. Visión de alumnos y profesores de la escuela secundaria argentina. Buenos Aires: Santillana.

Dussel, I. (2011). Aprender y enseñar en la cultura digital. VII Foro Latinoamericano de Educación. Experiencias y aplicaciones en el aula. Aprender y enseñar con nuevas tecnologías. Buenos Aires. Santillana. http://www.oei.org.ar/7BASICOp.pdf

Felicitas, A. (2012). La escuela secundaria argentina en perspectiva histórica y comparada: modelos institucionales y desgranamiento durante el siglo XX. Cadernos de História da Educação, 11(1), 131-144. Recuperado de http://www.seer.ufu.br/index.php/che/article/view/17534

Garrison, D. R. y Anderson, T. (2005). El e-learning en el siglo XXI. Investigación y práctica. Barcelona: Octaedro.

Grimson, A. (2011). Los límites de la cultura. Crítica de las teorías de la identidad. Buenos Aires: Siglo $\mathrm{XXI}$.

Kincheloe, J. y McLaren, P. (2012). Replanteo de la teoría crítica y de la investigación cualitativa. En N. Denzin e I. Lincoln, Paradigmas y Perspectivas en disputa (pp. 241-315). Barcelona: Gedisa.

Koselleck, R. (1993). Futuro pasado. Para una semántica de los tiempos históricos. Barcelona: Paidós.

Levy, D. (2013). De la red al aula. ¿Una nueva ilusión? En D. Korinfeld, D. Levy y S. Rascován (Eds.), Entre adolescentes, jóvenes y adultos. Puntuaciones de época (pp. 123-151). Buenos Aires: Paidós.

Martuccelli, D. (2009). La autoridad en las salas de clase. Problemas estructurales y márgenes de acción. Diversia, 1. Recuperado: de http://www.uff.br/observatoriojovem/sites/default/files/documentos/Martuccelli La autoridad en las salas de clase Diversia.pdf

Nobile, M. (2011). Redefiniciones de la relación docente-alumno: una estrategia de personalización de los vínculos. En G. Tiramonti (Dir.), Variaciones sobre la forma escolar. Límites y posibilidades de la escuela media ( pp. 179-204). Rosario, Arg.: Flacso- HomoSapiens.

Rascován, S. (2013). Entre adolescentes, jóvenes y adultos. En D. Korinfeld, D. Levy y S. Rascován (Eds.), Entre adolescentes, jóvenes y adultos. Puntuaciones de época (pp. 25-49). Buenos Aires: Paidós. 
Rockwell, E. (2009). La experiencia etnográfica. Historia y cultura en los procesos educativos. Buenos Aires: Paidós.

Sancho, J. (2010). Innovación, cambio y mejora en la enseñanza universitaria. Lo que añaden y lo que ocultan las TIC. En J. Paredes y A. De la Herrán (Coords.), Cómo enseñar en el aula universitaria. Madrid: Pirámide.

Skliar, C. (2012). La crisis de la conversación de alteridad. Educar entre generaciones. En M. Soutthwell (Comp.), Entre generaciones. Exploraciones sobre educación, cultura e instituciones (pp. 87-102). Rosario, Arg.: Flacso-HomoSapiens.

Southwell, M. (2013). La escuela ante nuevos desafios: participación, ciudadanía y nuevas alfabetizaciones. Buenos Aires: Santillana.

Tiramonti, G. (Dir.) (2011). Variaciones sobre la forma escolar. Límites y posibilidades de la escuela media. Rosario, Arg.: Flacso-HomoSapiens.

Tenti, E. (2005). La condición docente. Análisis comparado de la Argentina, Brasil, Perú y Uruguay. Buenos Aires: Siglo XXI-IIPE/UNESCO.

Tenti, E. (Comp.) (2006). El oficio de docente. Voacación, trabajo y profesión en el siglo XXI. Buenos Aires: Siglo XXI- IIPE/UNESCO.

Terigi, F., Briscioli, B., Scavino, C., Morrone, A. y Toscano, A. (2013). La educación secundaria obligatoria en la Argentina: entre la expansión del modelo tradicional y las alternativas de baja escala. Revista del IICE, 33, 27-46.

UNICEF Argentina-Ung (2012). Adolescentes y secundaria obligatoria. Finalización de estudios y vuelta a la escuela. Recuperado de www.unicef.org/argentina/spanish/FINES OKb.pdf

Vezub, L. (2011). ¿Qué cuentan las trayectorias de desarrollo profesional de los docentes sobre su oficio? En A. Alliaud y D. Suárez, El saber de la experiencia. Narrativa, investigación y formación docente (pp. 159-199). Buenos Aires: FFYL, UBA-CLACSO.

Ziegler, S. (2011). Entre la desregulación y el tutelaje: ¿hacia dónde van los cambios en los formatos escolares? En G. Tiramonti (Dir.), Variaciones sobre la forma escolar. Límites y posibilidades de la escuela media (pp. 71-88). Rosario, Arg: FLACSO. 